\title{
BMJ Open Enriching medical trainees' learning through practice: a video reflexive ethnography study protocol
}

\author{
Christy Noble, ${ }^{\oplus 1,2,3}$ Stephen Billett, ${ }^{4}$ Joanne Hilder, ${ }^{1}$ Andrew Teodorczuk, ${ }^{2}$ \\ Rola Ajjawi ${ }^{\circ}$
}

To cite: Noble C, Billett S, Hilder J, et al. Enriching medical trainees' learning through practice: a video reflexive ethnography study protocol. BMJ Open 2019;9:e031577. doi:10.1136/ bmjopen-2019-031577

- Prepublication history and additional material for this paper are available online. To view please visit the journal (http:// dx.doi.org/10.1136/bmjopen2019-031577).

We presented some of these methodological ideas during a Symposium - Learning about workplace learning: can video research methods help? - at the Australian and New Zealand Association of Health Professions Educators (ANZAHPE) conference 1st to 4th July, 2018 in Hobart, Australia.

Received 10 May 2019 Revised 11 July 2019 Accepted 24 July 2019

Check for updates

(C) Author(s) (or their employer(s)) 2019. Re-use permitted under CC BY-NC. No commercial re-use. See rights and permissions. Published by BMJ.

For numbered affiliations see end of article.

Correspondence to

Dr Christy Noble;

c.noble@griffith.edu.au

\section{ABSTRACT}

Introduction Supporting medical students' and junior doctors' development in busy clinical settings is challenging. As opportunities for developing trainees, for example, traditional bedside teaching, are decreasing, teaching outside of clinical practice is increasing. However evidence suggests that effective learning through practice arises via an interplay between, first, what experiences are afforded by clinical settings and, second, how trainees engage with these affordances. Many studies investigating clinician learning through practice focus on only one of these two factors. Yet, a well-recognised methodological challenge of enabling learners to articulate how and what they are learning through practice exists. We need, therefore, to understand how this relationship plays out in practice in ways that enrich learning.

Methods and analysis This protocol describes a video reflexive ethnographic approach to illuminate how learning through practice in hospital settings occurs and can be enriched. The study will be conducted in two phases. In phase I, senior clinicians from emergency medicine, medicine and surgical specialties will be interviewed about how they guide trainees' learning through practice. These forms of guidance, analysed using the framework method, will inform phase II comprising observations of practice in: (1) emergency, (2) medical and (3) surgical departments. Video recorded episodes of clinicians' guiding learning through practice will be shared and appraised in reflexive sessions with each clinical team. Relational interdependent learning theory informs the design and data analyses to elicit and evaluate strategies for guiding learning through practice.

Ethics and dissemination Ethical approval has been received from both healthcare and university settings. The findings should provide important insights for clinicians about workplace learning practices. Findings will be disseminated across the project phases and to diverse audiences-locally, nationally and internationally. The dissemination strategy will use seminars, grand rounds, conference presentations and academic papers to articulate practical, theoretical and methodological findings.

\section{INTRODUCTION}

Learning through everyday clinical work is established as an important contribution to medical education. ${ }^{12}$ This is hardly surprising because '...workplace learning is as old as

\section{Strengths and limitations of this study}

- Use an innovative approach-video reflexive ethnography (VRE) - to explore the enrichment of learning through practice in a traditionally challenging area of investigation.

- Engage clinical team members and patients in individual and collective learning experiences.

- Be informed by relational interdependent learning theory to assist with identifying the interdependence between learner engagement and workplace affordances.

- Generate pragmatic, immediate and potentially transformative solutions for enriching learning in work-intense clinical settings.

- Be limited to a single hospital setting and three clinical areas (ie, emergency medicine, medicine and surgery).

medicine itself...' (p15) ${ }^{3}$ and is recognised as making essential contributions to medical practitioners' initial and ongoing clinical development and identity formation. The demands of clinical practice are, however, becoming more intense ${ }^{4}$ leading to concerns about the viability of clinical teaching activities in and during clinical work (eg, bedside teaching) and the associated impact on clinical capabilities as a result of learning experiences being divorced from practice settings. ${ }^{5-7}$

The efficacy of learning through practice is founded on it occurring through engagement in authentic activities that can be sequenced and be supported through close guidance. $^{8} 9$ Evidence supports viewing learning through practice as an interdependent process, occurring through interactions between: (1) individual's engagement and (2) affordances of the clinical setting. ${ }^{8}$ Thus, illuminating and examining the relations between these two factors offers insights about supporting and enriching learning in busy clinical settings.

Currently, explorations of these interdependent processes (ie, the relations between individual cognition and influence of social 
settings) are largely absent in the literature. However, a well-recognised methodological challenge is for learners to articulate how and what they are learning in practice $^{9-11}$ This is because learning in practice is often 'largely invisible' as these experiences can be taken for granted or not acknowledged as learning. ${ }^{910}$ Also, the learning outcomes may not be immediately captured or result in observable behaviour change. Outcomes may be tacit or just seen as 'part of a person's general capability, rather than something that has been learned' (p249). ${ }^{10}$ The following section proposes a means for addressing this methodological challenge.

\section{Learning in healthcare contexts}

Healthcare settings provide or afford trainees rich opportunities for learning. Learning is supported by: (1) engaging in clinical activities and interacting with others, (2) observing others and (3) guidance from more experienced practitioners. ${ }^{812}$ Yet, healthcare settings vary considerably although by location (urban/rural) or specialty and offer diverse learning opportunities requiring different strategies to support learning. When considering how to effectively support learning through practice, these differences need to be accommodated. This adds further challenges as clinical contexts are described as 'dark matter', that is, 'something that we cannot touch, measure or see' (p808).$^{13}$ At the same time, broader societal factors such as shorter patient stays, constrained financial resources, increasing medical student and trainee numbers, technology innovations combined with the growing burden of disease compound this challenge. Moreover, clinicians are increasingly required to perform tasks other than patient care, including supervision and management roles while trying to educate in clinical settings. ${ }^{14}$

A further challenge, is providing meaningful patient engagement in these learning episodes in ways that can improve patient relationships with clinicians and the quality of trainee development. ${ }^{15}$ While, it is common for all three members of the triad-trainee, patient and supervisor-to be present during learning interactions, the involvement of patients can often be passive, that is, 'objectified as learning resources' (p918). ${ }^{16}$ A response to these challenges has been to use teaching activities outside of clinical practice such as 'protected teaching time' and continuous professional development (CPD). ${ }^{7}$ While helpful, this response fails to address the balancing of service commitments with the supervision of junior doctors and medical students remains, ${ }^{717}$ and through clinical experiences.

In justifying the methodological approach, it is helpful to consider workplace learning as two co-occurring activity systems: delivering patient care and enhancing trainee learning. This co-occurrence can cause tensions and contradictions, on the one hand, but also realise effective and synergistic outcomes for both care and learning, on the other. ${ }^{18}$ How that co-occurrence can be rendered effective is dependent on the people present, the social, cultural and material resources afforded by the situation. ${ }^{8}$ Much of this co-occurrence can be captured and appraised through video reflexive ethnography $(\mathrm{VRE})^{1920}$ that enables observation of these workarounds, and how work and learning co-occur across multiple people, places, objects and times through various complex assemblages. Thus, we focus on how workplace learning is optimised (or hindered) and for mobilising resources for optimising learning. Furthermore, through clinicians and trainees reviewing video excerpts, their practical expertise and wisdom can be harnessed to collaboratively identify learning and enrich working and learning.

The use of VRE in healthcare settings to redesign practice has increased since its inception in 2002, now exploring team communication, clinical handover, and ward-round redesign and infection control. ${ }^{21}$ Recently, it has been successfully used to understand and change health professions education, in areas as diverse as leadership practice, ${ }^{22}$ feedback ${ }^{23}$ and error recognition. ${ }^{24}$

\section{Objectives and research questions}

Our study aims to critically examine how medical learning through practice in hospital settings is occurring and can be enriched. We seek to examine how dyadic (ie, learners and clinical supervisors) and triadic (ie, patients, learners and clinicians) interactions are contributing to that learning. The specific research questions to be addressed are:

1. How do clinician educators balance patient service and medical education within the demands of clinical practice settings?

2. How do learning affordances of clinical settings shape medical trainee engagement?

3. How can learning through practice be enriched in busy clinical settings?

4. How can VRE be used to enrich clinical learning and patient care in sustainable and efficient ways?

\section{METHODS AND ANALYSIS}

\section{Study setting}

This research will be enacted in a healthcare service in South East Queensland, Australia. It will focus on three clinical areas: (1) emergency department, (2) medicine and (3) surgery. These areas have been selected as all medical students and junior doctors are required to complete placements or terms, respectively, in them. The healthcare service comprises of two hospitals and over 15 community centres and services. The smaller hospital is $<15$ years old and the large tertiary hospital was built in 2013 beside a publicly funded university.

\section{Design}

Interviews and VRE methods will be used to investigate the everyday practices of guiding learning in the three settings focusing on triadic interactions among trainees, supervisors and patients. ${ }^{20}{ }^{25}$ With VRE, researchers collaborate with participants, in our case medical staff, to make everyday practices visible, facilitate recall of sense-making and assist with generating strategies for improving learning in clinical settings. ${ }^{20}{ }^{25}$ Through VRE and associated interviews, 
everyday practice will be illuminated by combining outsider's perspective with insider's knowledge. As an interventionist research method, we aim to foster learning and change related to enriching learning through practice by creating opportunities, using visual data, for medical staff to question and, potentially, disrupt their taken-for-granted learning practices.

A key principle guiding this VRE study is 'exnovation', that is, the researchers in collaboration with the clinicians attempting to reveal and enhance existing practices, thereby making visible the taken-for-granted aspects of practice ${ }^{26}$ This is achieved by: (1) sensitising clinicians to their work learning practices through observing videos of them practicing; (2) the researchers gathering ideas and insights from clinicians through reflexive sessions; and (3) together, generating or coconstructing ideas for enriching practice based on their new learnings and insights. ${ }^{19}$

\section{This investigation will be conducted through two overlapping phases \\ Phase l: practitioner interviews}

Practitioner interviews comprises semistructured interviews with senior clinicians from three specialty groups, that is, emergency medicine, medicine and surgery. The purpose is to provide accounts and understandings of how learning through practice is enacted from the clinicians' perspectives and to provide insider knowledge for honing observations in phase II. The interviews will also be used to establish rapport and provide opportunities for recruitment of phase II participants.

\section{Phase II: video reflexive ethnography}

The VRE phase will be conducted with the three specialty groups and in two stages. First, in each clinical specialty, using ethnographic methods, we will observe everyday clinical practice and/or participants' accounts of healthcare, and in our case, processes that support medical trainee learning through practice episodes within the dyad and triad. These episodes will be videoed. Second, the videos will be edited to provide excerpts that capture triadic and dyadic interactions among and between informants. The participants will then review edited video footage in small groups to offer perspectives on the working-learning events and discuss and share their perspectives on what is occurring in these events and for what purposes. This approach will assist with identifying taken-for-granted practices. The focus of these reflections is prospective (eg, 'what does this feedback make possible') rather than retrospective (eg, 'what is going on'). ${ }^{20}$ That is, to identify how these interactions might serve to promote more effective working-learning in clinical settings. These data will be used to generate suggestions for improving practice that will be subsequently appraised by the informants in a coconstructive process. This appraisal will, first, consider the practicalities associated with these suggestions and render them as working principles. Second, the appraisal will support the prospects of these principles to be translatable to practice.

\section{Theoretical framework}

This research method is informed by relational interdependent learning theory. ${ }^{27}$ From this perspective, learning is considered as a process reliant on mutual contributions between learners' individual agency (eg, intentionality, subjectivity and identity) and the social experience (eg, workplace norms, practices and values). ${ }^{27}$ The key premise is that neither the contributions and suggestions of social experience (eg, clinical setting), nor individuals' knowledge, knowing and agency (ie, what they know, can do and value) alone is sufficient to promote effective occupational learning. ${ }^{27}$ Instead, it is the interdependence between these contributions that is salient.

\section{Patient and public involvement}

While the study will focus on triad interactions, this protocol paper was developed through engaging medical practitioners and students, but not patient involvement. Patients were not invited to comment on the study design or to contribute to the writing or editing of this document for readability or accuracy.

\section{Participants}

\section{Phase I: practitioner interviews}

Practitioner interviews participants will be senior doctors working in emergency medicine, medicine and surgical settings. Senior doctors here are defined as consultants/fellow or registrars. Approximately 15-20 senior doctors working in emergency medicine, medicine or surgical setting at the healthcare service will be interviewed. To understand ${ }^{28}$ how clinicians enrich learning through practice while balancing clinical commitments, a two-staged approach to sampling will be used. First, maximum variation sampling will be used to elicit experiences of enriching learning through practice from a diverse group of senior doctors. ${ }^{29}$ Table 1 provides an overview of proposed sampling framework.

Second, to supplement the sample identified through the first stage, snowball sampling ${ }^{29}$ will be used. That is, interview participants being asked to identify other clinicians who effectively enrich learning through practice. It is anticipated that each interview will take between 30 and $45 \mathrm{~min}$. Recruitment will be conducted to achieve sufficiency, that is, enough to enable transferability of findings across clinicians and richly answering the research questions and to inform Study $2 .^{30}$

\section{Phase II: video reflexive ethnography}

In this phase, two groups of participants: (1) medical staff (including medical students) from: emergency department, medicine and surgery; and (2) patients under the care of these medical staff. Initially, we will recruit at least one medical team from each clinical setting. A medical team usually includes consultants and fellows $(n=1-2)$, registrar $(n=1-2)$, junior doctors and/or interns $(n=1-2)$ and medical students $(n=1-2)$. In sum, between 25 and 50 medical practitioners, with representation from a range of levels, will participate. 


\begin{tabular}{ll}
\hline $\begin{array}{l}\text { Table } 1 \text { Propose sampling framework for senior clinician } \\
\text { phase I interviews }\end{array}$ & $\begin{array}{l}\text { Proposed no of } \\
\text { participants }\end{array}$ \\
\hline Criteria & 7 \\
\hline Clinical specialty & 7 \\
\hline Emergency medicine & 7 \\
\hline Medicine & 10 \\
\hline Surgery & 10 \\
\hline Senior doctors & $2-3$ \\
\hline Registrar & \\
\hline Consultant/fellows & 10 \\
\hline Formal educator role & 10 \\
\hline Gender & \\
\hline Female & 10 \\
\hline Male & 10 \\
\hline Years of experience & \\
\hline $5-10$ &
\end{tabular}

Then, for each medical team, the research team will recruit up to 10 patients and/or family members seen by the medical team.

\section{Sampling}

The sampling rationale is to be dyadic (eg, junior and senior doctor) and triadic (eg, patient, junior and senior doctor) learning encounters. For each medical team, these learning encounters (informed by our Study 1 interviews) will be sampled purposively, including (1) ward rounds, (2) handovers, (3) teaching of procedural skills, (4) bedside teaching encounters and (5) patient history taking will be videoed. There might be unanticipated spaces where learning encounters occur that will also be included. It is anticipated that at least three of each of these encounters, that is, up to 15 of them will be recorded, as with other VRE studies, ${ }^{31}$ to present diverse perspectives of these dyadic and triadic learning encounters, to generate rich reflexive discussions.

As it is exploratory and dialogic, VRE is a dynamic and emergent methodology making estimates of sample size difficult. ${ }^{19}$ Consequently, researchers will use their professional judgement to decide when sufficient examples of learning encounters have been sampled to enable effective reflexive discussions to occur.

\section{Inclusion criteria}

Participants will be over 18 years old, conversant in English, willing and able to give informed consent to participate, and for patients (and/or their substitute decision-makers (SDM) or other family members nominated by the patient), under the care of the clinicians who are willing to participate in phase II of the study.

\section{Recruitment}

\section{Phase l: practitioner interviews}

The research team will request that clinical directors at the site circulate information to medical consultants and registrars working in emergency department, medicine and surgery settings. Potential participants will be provided with the researchers' contact details and study information. The researchers will contact interested potential participants and inform them about the research procedures, including their consent. Verbal consent will be obtained from participants at the commencement of the interview.

\section{Phase II: video reflexive ethnography}

For medical staff participants, the researchers will first meet with the key medical contacts in the three clinical areas. These key contacts and champions will be provided with a participant information sheet about the study and the researchers' contact details. Their endorsement will be sought. When this has been received, they will be asked to distribute the participant information sheet to their teams and colleagues. Also, permission will be sought to present information about the study (including descriptions about VRE, what is involved and explanation of the consent process) within team meetings. Potential participants will have 1 week to decide if they will participate in the study. For teams agreeing to participate, the researchers will also meet with the nurse unit managers working within the clinical settings where the medical teams work and provide them with study information.

Second, patient participants will be those being treated by the medical team and will be identified by the medical team. Potential patient participants will be approached directly by the field researcher and invited to participate. The research team will invite patients and/or their SDM and/or other family members nominated by the patient to participate in the study. Patient participants who agree to participate will provide either written or audio recorded informed consent. Where clinical staff deem a patient to not have capacity to provide informed consent, the researcher/s will approach the patient's SDM to seek proxy informed consent. The potential participants will have up to 24 hours to decide if they would like to participate in the study. The study will adopt a continuous consent process ${ }^{19} 32$ described in detail in the Consent section.

Non-participants who coincidentally present during those observations potentially include other healthcare professionals (eg, nursing or allied health) or other patients. No identifying information will be collected relating to these groups and they will not be the focus of any observations.

\section{Consent}

\section{Phase I: practitioner interviews}

Verbal consent to participate in this study will be obtained prior to the interview which will be audio recorded.

Phase II: video reflexive ethnography

\section{Medical staff}

Medical staff indicating their interest in participating will be invited to provide written consent at the start of their 
involvement. However, throughout the data collection period, to ensure informed consent principles are met, the researchers will continue to request verbal consent during the VRE data collection, that is, before any period of observation, after videoing participants and before the reflexive sessions. ${ }^{33}$

\section{Patients and/or family members}

On recommendations from medical staff, patients (and/or SDM if the patient cannot consent) and/or family members will be invited to participate in the study and to give consent to having their clinical encounter video recorded and if acceptable, for a researcher to be present. Again, ongoing verbal consent will be requested, that is, before any period of observation or after videoing participants. ${ }^{33}$

For both phases, medical and patient participants may withdraw from the study at any time without any consequences. If a participant wishes to withdraw from the study, they are able to complete a revoke consent form. The researchers will then remove them from the study and delete their data. Should any individual who has not consented to participate be recorded, their image will be blurred, and their voice transcript deleted.

\section{Data collection}

\section{Phase l: practitioner interviews}

One-off, semistructured interviews will be conducted face-to-face and audio recorded. The interview guide (see online supplementary materials), informed by interdependent learning theory ${ }^{27}$ aims to explore clinicians' experiences of facilitating trainees' learning through practice. To enhance the data analyses, interviewers will take journal notes to capture observations and reactions at or just after the time of the interviews about their role and influence during the interviews. ${ }^{3435}$ Interviews will be deidentified and data professionally transcribed.

\section{Phase II: video reflexive ethnography}

Data in phase II will be gathered by two clinically-informed research team members whose role will include 'clinalyst', ${ }^{36}$ that is, an 'outsider-analyst-catalyst' (p176). They also work in the study hospital as a research fellow and research officer, thus could be considered as insiders, yet do not work day to day with clinical staff, so will also have outsider perspectives to catalyse insiders' knowledge through sharing videos and promoting reflection on practice. ${ }^{36} 37$

VRE data collection will occur in three clinical settings-emergency department; medicine and surgery-and be conducted in three sequential stages: (1) field observations; (2) video recording; (3) reflexive sessions. This data collection will coincide with clinical activities and as guided and advised by the lead medical practitioner. It is anticipated that each VRE cycle will take between 3 and 4 months.

\section{Field observations}

The researchers will engage in general ethnographic observations prior to video data collection to build trust between the researcher and participants and to identify teachable interactions and moments. ${ }^{22} 253839$ The field researchers will shadow the medical team as they engage in their day to day activities observing dyadic and triadic interactions. These researchers will generate field notes about the types of interactions occurring, when they occur, in what setting and timeframes for interactions and will be coded and discussed with the research team. These observations, combined with findings from Study 1 interviews, will assist the researchers to understand the field of study and areas of focus for the video recordings while building informant rapport.

\section{Video recording}

Once the medical teams and patients agree for their activities to be videoed, the recording phase will begin. Based on other VRE studies, ${ }^{20} 254041$ this timeframe can vary between a few days to weeks, yet is dependent on when the team and patients agree that the video recordings can commence. As noted above, it is anticipated that at least 15 episodes of dyadic and/or triadic encounters will be recorded and is likely to take several days, again, based on other VRE studies, but these are only estimates. ${ }^{20}{ }^{25}$

\section{Reflexive sessions}

The researchers will select recorded sections illustrating dyadic and triadic interactions observed in phase II. The selection of the video clips will be based on the following criteria (informed by Iedema $e t a l^{19}$ ): level of detail; clear visibility; day-to-day or exceptional examples of enriching learning; diversity of learning activities. Also, ethical judgement will guide clip selection to ensure inappropriate or problematic clips, such as, existing tensions or dynamics within the team are not used and before sharing clips, individuals who are in the clips will be asked to approve their sharing. ${ }^{2025}$

During the reflexive sessions, participants will be shown three to four short video clips between 2 and $4 \mathrm{~min}$, as in other VRE studies in hospital settings. ${ }^{20} 254041$ It is anticipated that these sessions will last between 60 and $90 \mathrm{~min}$ and be audio recorded and transcribed. Where there are issues related to power or hierarchy, the researchers will offer separate reflexive sessions. For example, the medical students and junior doctors may be offered separate reflexive sessions to alleviate potential concerns about power. See online supplementary materials for an overview of the reflexive session structure and questions.

\section{Data management}

All data, transcripts and videos, will be stored electronically on a secure shared drive that is subject to institutional requirements for data security. Paper documents will be stored in a locked cabinet. Data will be stored for a minimum of 5 years after publication of project findings and then all data obtained through this study will be destroyed, for example, permanent deletion of any electronic data files or audiotapes. Any hard copy material will be shredded and disposed of in the confidential waste system. 


\section{Data analysis}

Phase I: practitioner interviews

The data analysis will follow the framework method described by Ritchie and Spencer ${ }^{42}$ and will be conducted in five stages: (1) familiarisation; (2) identifying a thematic framework (informed by interdependent learning theory); (3) indexing; (4) charting and (5) mapping and interpretation. Practically, interviews will be transcribed and uploaded to NVivo and research teams will each read the transcripts to identify participants' interpretations and constructs. ${ }^{43}$ To identify a thematic coding framework, a subset of the transcripts will be reviewed in-depth and coded. Based on this subset coding, a preliminary coding framework will be developed through discussion among the research team while being informed by our theoretical framework of relational interdependence. ${ }^{27}$ The entire dataset will be coded using the team-negotiated framework. Through this process, codes will be grouped into subthemes and similar codes collapsed which will be further elaborated and their relationships clarified by reading and rereading all data. The findings from each interview will then be compared across the interviews, focusing on areas of consensus and difference, with these findings providing evidence for strategies to enrich learning through practice while balancing clinical commitments. Key to qualitative analyses are the discussions and meaning making within the team whose members possess distinct theoretical and methodological perspectives. The team comprises: two health professions education researchers with backgrounds in hospital pharmacy (CN) and physiotherapy (RA), respectively; a medical education researcher/consultant psychiatrist (AT); a workplace learning researcher with extensive experience in healthcare education research (SB); and a research trainee with a social work background $(\mathrm{JH})$.

\section{Phase II: video reflexive ethnography}

Data will take the form of field notes, and audio and video recordings. First, the research team will edit footage into narrative governed scenes deemed to be representative, based on their observations, of common learning through practice episodes including dyadic and triadic interactions. Footage will be edited using iMovie and segments selected according to these common practices and shown at reflexive feedback sessions for further critique and analysis by the participants and researchers. In this way, visual material reflexively viewed by participants produces a further layer of knowledge of how learning is enriched through practice. Moreover, this approach aligns to our theoretical framework, relational interdependent learning theory, ${ }^{27}$ whereby both individual engagement and cognition is being explored in relationship to the social suggestions of clinical practice. In keeping with VRE, the reflexive meetings form the basis of the data analysis focusing on the participants' reflections and meanings, and the research team, given to images in different contexts and examining how visual content is informed by the subjectivities and intentions of the individuals involved.
Based on VRE methodology, field notes and audio recordings from the reflexive sessions will be analysed to identify how medical learning through practice in hospital settings is being enriched by facilitating meaningful dyadic and triadic engagement and collaborations. This data analysis will follow the framework method ${ }^{42}$ that comprises the five stages described above. This analytical approach has been using in other VRE studies. ${ }^{2344}$ The analysis informed by our theoretical framework-will focus on the interplay between the learners' agency (ie, how they choose to engage with activities and interactions) and the learning affordances (ie, day-to-day clinical practice).

\section{Limitations}

There are potential limitations with this study design. First, the study will be conducted in a single hospital; however, three discrete settings will be investigated thus likely to provide an enhanced degree of transferability. Second, we are focusing phase I interviews on senior clinicians to identify strategies being used to enrich learning through practice. These strategies will inform sampling for phase II where trainee and patient experiences and insights will be included. Third, concerns might be raised about the Hawthorne effect, that is, the effect where participants alter their behaviour when being observed. However, considerable evidence suggests that this effect in observational research is small. ${ }^{45}$ Indeed, other VRE studies have found that the effect is small and short-lived. ${ }^{202546}$ This is because it is challenging for practitioners to sustain modified behaviour to which they have been habituated. A formal evaluation of the VRE method found that participants became accustomed to the researcher presence and ignored the camera. ${ }^{47}$ Moreover, the practical and far reaching benefits of observing one's own practice go beyond concerns about the Hawthorne effect. ${ }^{20}$

\section{Outcomes}

Given the transformative nature of VRE, the study's key outcome is expected to be improved learning through practice within triadic interactions. The proposed research aims to generate the following outcomes.

\section{Enrich learning through practice and contribute to improved medical students' and junior doctors' learning and patient outcomes}

Learning through practice, when guided by experts, is a key pedagogical practice in healthcare. Effective supervision develops competent medical trainees, who are central to safe and effective healthcare, while improving learning and patient outcomes. ${ }^{48}$ Improved efficiency for medical staff will be realised through aligning patient care with effective workplace learning by privileging the triadic relationship. Moreover, improving learning through practice contributes to enhanced medical trainee work satisfaction, trainee well-being and improved retention. ${ }^{49}$ Lastly, VRE will create an opportunity for clinicians and trainees to reflect on their own practice and the taken for granted tensions related to learning through practice. 
Contribute to new knowledge about how practitioners enrich learning through clinical practice

VRE methodology can bring changes practice through both empowering staff and highlighting effective learning approaches through clinical work. Strategies and guidelines, coproduced with the researchers, will likely influence practice in more effective and credible ways that foreground voices often not heard in supervisor development programme. In particular, strategies for balancing care while facilitating learning will be identified.

\section{Contextualise workplace learning principles}

It is anticipated that by applying relational interdependent learning theory to VRE methodology we will meaningfully contextualise the principles of workplace learning for clinicians. This will enable clinicians to consider workaround strategies when experiencing the tensions balancing patient care and supporting trainee learning. To date, these strategies have largely been based on self-reported data.

\section{Patient benefits}

By including, illuminating and examining the patient experience of engaging in triadic learning interactions about, practical strategies for and insights can be gained on how patients may engage in these interactions to support their learning about their care. This is important because the exclusion of patients in medical education research has been identified as a missed opportunity to make essential contributions to better preparing clinicians for ever-changing patient needs. ${ }^{50}$

\section{Knowledge translation}

First, research practice gaps often exist. Despite the importance of effective learning through practice to doctor capability and patient outcomes, evidence-based guidelines informing this practice are sparse and/or tend to focus on teacher-lead/educational opportunities. ${ }^{7}$ This study will identify the key features and factors influencing learning through practice and will inform a tailored, theory-informed intervention. Second, VRE is recognised as both a research and practice development methodology. ${ }^{51}$ Thus, the knowledge will be co-produced between researchers and participant $\mathrm{s}^{52}$ and through these interactions contributing to the remaking of learning through practice culture. ${ }^{53}$ Finally, our findings will enable us to construct learning vignettes to support clinicians' and trainees' development about more effectively engaging in learning through practice.

\section{Policy contributions}

Evidence is lacking to inform policy addressing effective learning through clinical practice. This research will provide much needed guidance for healthcare organisations to support effective learning through day-to-day work activities.

\section{ETHICS AND DISSEMINATION}

We received ethical approval from Gold Coast Health for phase I (HREC/18/QGC/32) on 23rd February 2018 and phase II (HREC/18//QGC/131) on 5th June 2018 and site-specific management and approval for phase I (SSA/18/QGC/97) on 20 April 2018 and phase II (SSA/18/ QGC/133) on 17 July 2018. Because the study will involve medical students, we also received full ethics approval from Griffith University (2018/854) on 11 November 2018. Findings will be disseminated across the project phases and in distinct ways to diverse audiences-locally, nationally and internationally. Combination of seminars, conference presentations and academic papers will be used to articulate practical, theoretical and methodological findings.

\section{Glossary}

Affordances

The invitational qualities of a workplace for learning. ${ }^{54}$

Continuous professional development (CPD)

The learning activities professionals engage in to develop and enhance their abilities. It enables learning to become conscious and proactive, rather than passive and reactive'.

\section{Reflexive sessions}

Sessions where selected video footage are played back to participants 'to allow them to view their own practices from a different perspective' (p58). ${ }^{19}$

\section{Video reflexive ethnography}

'A method that combines ethnography (the observation and analysis of practice) with the use of video as a learning tool. This method gives front-line staff the opportunity to reflect on their everyday practices, using video footage of those practices' (p58). ${ }^{19}$

\section{Author affiliations}

${ }^{1}$ Allied Health and Medical Education Unit, Gold Coast Hospital and Health Service, Southport, Queensland, Australia

${ }^{2}$ School of Medicine, Griffith University, Gold Coast, Queensland, Australia ${ }^{3}$ School of Pharmacy, The University of Queensland, St Lucia, Queensland, Australia ${ }^{4}$ School of Education and Professional Studies, Griffith University, Mount Gravatt, Queensland, Australia

${ }^{5}$ Centre for Research in Assessment and Digital Learning, Deakin University, Melbourne, Victoria, Australia

Twitter@chnoble@HilderJoanne @r_ajjawi @andyteodorczuk @StephenBillett3

Acknowledgements We are grateful for the guidance and methodological expertise provided by Professors Charlotte Rees, Victoria Brazil and Dr Aileen Collier. We presented some of these methodological ideas during a SymposiumLearning about workplace learning: can video research methods help? - at the Australian and New Zealand Association of Health Professions Educators (ANZAHPE) conference 1 to 4 July 2018 in Hobart, Australia.

Contributors CN, AT, RA, SB and JH were responsible for the project conception. $\mathrm{CN}$ led the protocol development and prepared the first draft of this manuscript. AT, $\mathrm{RA}, \mathrm{SB}$ and JH developed and refined the study protocol. All authors provide critical evaluation and revisions of the manuscript and have read and approved the final manuscript.

Funding This project is funded by the Gold Coast Health Collaborative Research Scheme-RGS2017-LG0002.

Competing interests None declared.

Patient consent for publication Not required. 
Ethics approval Institutional ethics approval was obtained from the study site and relevant universities.

Provenance and peer review Not commissioned; externally peer reviewed.

Open access This is an open access article distributed in accordance with the Creative Commons Attribution Non Commercial (CC BY-NC 4.0) license, which permits others to distribute, remix, adapt, build upon this work non-commercially, and license their derivative works on different terms, provided the original work is properly cited, appropriate credit is given, any changes made indicated, and the use is non-commercial. See: http://creativecommons.org/licenses/by-nc/4.0/.

\section{REFERENCES}

1. Australian Medical Council. National framework for medical internship, 2019. Available: https://www.amc.org.au/accreditationand-recognition/assessment-accreditation-prevocational-phasemedical-education/national-internship-framework/ [Accessed $17 \mathrm{Apr}$ 2019].

2. Weiss KB, Bagian JP, Nasca TJ. The clinical learning environment: the foundation of graduate medical education. Jama-j Am Med Assoc 2013;309:1687-8

3. Dornan T, learning W. Workplace learning. Perspect Med Educ 2012;1:15-23.

4. Plsek PE, Greenhalgh T. Complexity science: the challenge of complexity in health care. BMJ 2001;323:625-8.

5. Crumlish CM, Yialamas MA, McMahon GT. Quantification of bedside teaching by an academic hospitalist group. J Hosp Med 2009;4:304-7.

6. Mikhail M.The death of bedside teaching The BMJ Opinion The BMJ; 2016.

7. Sholl S, Ajjawi R, Allbutt $\mathrm{H}$, et al. Balancing health care education and patient care in the UK workplace: a realist synthesis. Med Educ 2017;51:787-801.

8. Billett S. Learning through health care work: premises, contributions and practices. Med Educ 2016;50:124-31.

9. Teunissen PW, Experience TPW. Experience, trajectories, and reifications: an emerging framework of practice-based learning in healthcare workplaces. Adv Health Sci Educ Theory Pract 2015;20:843-56.

10. Eraut * M. Informal learning in the workplace. Studies in Continuing Education 2004;26:247-73.

11. Teodorczuk A, Ajjawi R, Billett S, et al. The service/teaching tension: a window into the soul of a hospital. Med Educ 2018;52:678.

12. Varpio L, Bidlake E, Casimiro L, et al. Resident experiences of informal education: how often, from whom, about what and how. Med Educ 2014;48:1220-34.

13. Bates J, Ellaway $\mathrm{RH}$. Mapping the dark matter of context: a conceptual scoping review. Med Educ 2016;50:807-16.

14. Westerman M, Teunissen PW, van der Vleuten CPM, et al. Understanding the transition from resident to attending physician: a transdisciplinary, qualitative study. Academic Medicine 2010;85:1914-9.

15. Wong BM, Holmboe ES. Transforming the academic faculty perspective in graduate medical education to better align educational and clinical outcomes. Academic Medicine 2016:91:473-9.

16. Monrouxe LV, Rees CE, Bradley P. The construction of patients' involvement in hospital bedside teaching encounters. Qual Health Res 2009;19:918-30.

17. Sheehan D, Jowsey T, Parwaiz M, et al. Clinical learning environments: place, artefacts and rhythm. Med Educ 2017;51:1049-60.

18. Ajjawi R, Rees C, Monrouxe LV. Learning clinical skills during bedside teaching encounters in general practice: a video-observational study with insights from activity theory. Journal of Workplace Learning 2015;27:298-314.

19. ledema R, Carroll K, Collier A, et al. Video-Reflexive ethnography and healthcare improvement: theory and application. Boca Raton, FL: CRC Press, Taylor \& Francis Group, 2019.

20. ledema R, Mesman J, Carroll K. Visualising healthcare practice improvement: innovation from within. London: Radcliffe, 2013.

21. ledema R, Hor S-Y, Wyer M, et al. An innovative approach to strengthening health professionals' infection control and limiting hospital-acquired infection: video-reflexive ethnography. BMJ Innovations 2015;1:157-62.

22. Gordon L, Rees C, Ker J, et al. Using video-reflexive ethnography to capture the complexity of leadership enactment in the healthcare workplace. Adv Health Sci Educ Theory Pract 2017;22.

23. Urquhart LM, Ker JS, Rees CE. Exploring the influence of context on feedback at medical school: a video-ethnography study. Adv Health Sci Educ Theory Pract 2018;23.
24. Gough S, Yohannes AM, Murray J. Using video-reflexive ethnography and simulation-based education to explore patient management and error recognition by PRE-REGISTRATION physiotherapists. Adv Simul 2016;1.

25. Carroll K, ledema R, Kerridge R. Reshaping ICU ward round practices using Video-Reflexive ethnography. Qual Health Res 2008;18:380-90.

26. Mesman J. Uncertainty in medical innovation: experienced pioneers in neonatal care. London: Palgrave Macmillan UK, 2008.

27. Billett S. Relational interdependence between social and individual agency in work and working life. Mind, Culture, and Activity 2006;13:53-69.

28. Lincoln YS, Guba EG, Pilotta JJ, Inquiry N, et al. Naturalistic inquiry. Newbury Park, CA: Sage Publications, 1985: 9. 438-9.

29. Patton MQ. Qualitative research \& evaluation methods: integrating theory and practice. Thousand Oaks, California: SAGE Publications, Inc, 2015

30. Varpio L, Ajjawi R, Monrouxe LV, et al. Shedding the cobra effect: problematising thematic emergence, triangulation, saturation and member checking. Med Educ 2017;51:40-50.

31. McLeod HM. Respect and Shared Decision Making in the Clinica Encounter: A Video-Reflexive Ethnography [Ph. D.]. University of Minnesota 2017.

32. O'Reilly M, Parker N, Hutchby I. Ongoing processes of managing consent: the empirical ethics of using video-recording in clinical practice and research. Clin Ethics 2011;6:179-85.

33. Andrew C. Haunted by images? ethical moments and anxieties in visual research. Methodological Innovations Online 2013;8:68-81.

34. Watt D. On becoming a qualitative researcher: the value of reflexivity. The Qualitative Report 2007;12:82-101.

35. Enosh G, Ben-Ari A. Reflexivity: The Creation of Liminal Spaces-Researchers, Participants, and Research Encounters. Qual Health Res 2016;26:578-84

36. ledema R, Carroll K. The "clinalyst": Institutionalizing reflexive space to realize safety and flexible systematization in health care. Journal of Organizational Change Management 2011;24:175-90.

37. Carroll K, Mesman J. Multiple researcher roles in Video-Reflexive ethnography. Qual Health Res 2018;28:1145-56.

38. ledema R, Long $\mathrm{D}$, Forsyth $\mathrm{R}$, et al. Visibilising clinical work: video ethnography in the contemporary Hospital. Health Sociology Review 2006;15:156-68.

39. Pink S. Doing visual ethnography. Sage 2013.

40. Collier A, Phillips JL, ledema R. The meaning of home at the end of life: a video-reflexive ethnography study. Palliat Med 2015;29:695-702.

41. Hor S-Y, ledema R, Manias E. Creating spaces in intensive care for safe communication: a video-reflexive ethnographic study. BMJ Qual Saf 2014;23:1007-13.

42. Ritchie J, Spencer L. Qualitative data analysis for applied policy research. In: Bryman A, Burgess R, eds. Analyzing qualitative data. London: RoutledgeFalmer, 1994.

43. Tai J, Ajjawi R. Undertaking and reporting qualitative research. Clin Teach 2016;13:175-82.

44. Gordon L, Jindal-Snape D, Morrison J, et al. Multiple and multidimensional transitions from trainee to trained doctor: a qualitative longitudinal study in the UK. BMJ Open 2017;7:e018583.

45. Paradis E, Sutkin G. Beyond a good story: from Hawthorne effect to reactivity in health professions education research. Med Educ 2017:51:31-9.

46. ledema R, Merrick ET, Rajbhandari D, et al. Viewing the takenfor-granted from under a different aspect: a video-based method in pursuit of patient safety. Int J Mult Res Approaches 2009;3:290-301.

47. Manojlovich M, Frankel RM, Harrod M, et al. Formative evaluation of the video reflexive ethnography method, as applied to the physiciannurse dyad. BMJ Quality \&amp; Safety 2019;28.

48. Farnan JM, Petty LA, Georgitis E, et al. A systematic review: the effect of clinical supervision on patient and residency education outcomes. Acad Med 2012;87:428-42.

49. Lachish S, Goldacre MJ, Lambert T. Associations between perceived institutional support, job enjoyment, and intentions to work in the United Kingdom: national questionnaire survey of first year doctors. BMC Med Educ 2016;16:151.

50. Teodorczuk A, Yardley S, Patel R, et al. Medical education research should extend further into clinical practice. Med Educ 2017;51:1098-100

51. Collier A, Collier A, Collier A, et al. Practice development using video-reflexive ethnography: promoting safe space(s) towards the end of life in hospital. International Practice Development Journal 2016;6:1-16. 
52. Rycroft-Malone J, Burton CR, Bucknall T, et al. Collaboration and Co-Production of knowledge in healthcare: opportunities and challenges. Int J Health Policy Manag 2016;5:221-3.
53. Billett S, Smith R, Barker M. Understanding work, learning and the remaking of cultural practices. Studies in Continuing Education 2005;27:219-37.

54. Billett S. Workplace Pedagogic practices: Co-participation and learning. British Journal of Educational Studies 2002;50:457-81. 